\title{
DOS ESTRUCTURAS PARA EL PARTICIPIO PASADO
}

En este trabajo presento un primer avance de la investigación sobre el tema de la oposición concordancia/ausencia de concordancia en los participios pasados del español, italiano y francés ${ }^{1}$. El hecho de que la presente exposición esté orientada más-particularmente hacia el español, y contenga sólo algunos datos indicativos del italiano y del francés, es circunstancial: en realidad el análisis se llevó a cabo con base en los datos de las tres lenguas y tengo el propósito de completar la presentación del tema en otro artículo.

\section{Premisa}

En latín todo participio pasado (de ahora en adelante pp) tenía una concordancia nominal, o sea que concordaba con el elemento nominal al cual se refería por medio de oposiciones morfológicas de género, número y caso. En las lenguas romances actuales, en cambio, hay dos tipos diferentes de pp: el tipo que sigue manifestando concordancia nominal, por medio de las oposiciones morfológicas masculino/femenino y singular/plural, y un segundo tipo - que no existía en latín - que aparece siempre en la forma

${ }^{1}$ Ésta es una investigación que he empezado y adelantado durante un año sabático que disfruté en el Seminario di Linguistica e di Didattica delle Lingue de la Università degli Studi di Venezia, en el marco de un seminario de G. Cinque sobre la ergatividad en italiano. Allí tuve el privilegio de la orientación y la ayuda de G. Cinque, R. Kayne, G. Longobardi y L. Rizzi. Estoy muy profunda y sinceramente agradecida por el estímulo que recibí tanto de los argumentos que me convencieron como de aquéllos de los que disentí. La heterodoxia en el manejo de la teoría y en la forma de exponer es mi responsabilidad y se debe al hecho de que un año sabático, aun bisiesto, es demasiado corto. 
no marcada de masculino singular y por lo tanto no concuerda ni en género ni en número con ningún elemento nominal de la oración. Para el español esta situación está ejemplificada en las oraciones 1) y 2):

1) La novela fue traducida por Juana.

2) Juana ha traducido la novela.

Las primeras preguntas que surgen de esta observación son: ¿cuáles son las configuraciones que corresponden en la oración a los dos tipos de pp? ¿Cómo ha surgido el pp que carece de concordancia? Me parece que una respuesta satisfactoria a estas preguntas puede estar basada en la premisa de que el pp es un componente intrínsecamente categorizado en el léxico con el conjunto de rasgos $[+\mathrm{V} \pm \mathrm{N}]$. Los dos conjuntos de rasgos, $[+\mathrm{V}+\mathrm{N}]$ y $[+\mathrm{V}-\mathrm{N}]$, contrastan significativamente y la elección del uno o del otro acarreará consecuencias visibles en la sintaxis. El conjunto $[+\mathrm{V}+\mathrm{N}]$ corresponde a un elemento que tiene algún rasgo verbal pero no es plenamente y solamente un verbo; el conjunto $[+\mathrm{V}-\mathrm{N}]$ es un verbo, hasta es el verbo-tipo. Decir entonces que en las lenguas romances actuales el pp a veces es $[+\mathrm{V}+\mathrm{N}]$ y a veces $[+\mathrm{V}-\mathrm{N}]$ es solamente una manera distinta de decir que se trata de un elemento que tiene la característica de funcionar a veces como elemento verbal-nominal y a veces como elemento plenamente verbal. En el primer caso se trata de un elemento que está sometido a las propiedades de los nominales - y una propiedad muy peculiar de los nominales es la concordancia nominal, o sea la concordancia en género y número. En el segundo caso se trata de un elemento verbal, sometido a las propiedades del verbo - y el verbo tiene una concordancia de persona con su sujeto, pero carece de otro tipo de concordancia.

\section{El PARTICIPIO PASADO CON CONCORDANCIA NOMINAL}

En esta sección del trabajo expongo mi análisis del pp con concordancia nominal. Según la premisa anunciada, este pp tiene ras$\operatorname{gos}[+\mathrm{V}+\mathrm{N}]$. Considérense los ejemplos 3):

3) a. Entregados los equipajes, empezó la huelga.

b. Llegados los equipajes, empezó la huelga.

c. Tengo traducidos los resúmenes. 
d. Los equipajes fueron entregados por María.

e. María se casó drogada.

f. Juan encontró a María amarrada.

En todas estas oraciones hay un pp $[+\mathrm{V}+\mathrm{N}]$. Éste aparece, por lo menos, en frases participiales absolutas (3a y $3 \mathrm{~b}$ ); con el verbo tener (3c); con ser (3d); y en construcciones predicativas del sujeto y del objeto (respectivamente $3 \mathrm{e}$ y $3 \mathrm{f}$ ).

No he mencionado varios otros tipos de ocurrencias de pp con concordancia nominal, en particular los que están asociados con estar y los pp adjetivados que aparecen en sintagmas adjetivales: en este momento no pretendo abarcar exhaustivamente todos los casos de pp con concordancia nominal. Intento aquí, solamente, dar cuenta de las construcciones ejemplificadas en 3) de una manera unificada, por medio de un solo constituyente de características simples.

El constituyente que propongo es un "sintagma participial" (de ahora en adelante pp') cuyas características son las siguientes:

- su núcleo es un pp con rasgos $[+\mathrm{V}+\mathrm{N}]$ (por lo tanto sometido a concordancia nominal);

- este núcleo genera a su derecha un $\mathrm{SN}$, cuya presencia es obligatoria (algunas veces en forma de huella);

- siendo nominales ambos elementos, entre el núcleo y el SN generado a su derecha se establece concordancia nominal análogamente a como se establece la concordancia entre un sustantivo y un adjetivo;

- el sintagma nominal del pp' debe tener determinante;

- pp' puede tener expansiones; en particular acepta y hasta requiere - para tener mayor aceptabilidad - sintagmas adverbiales temporales y modales;

- pp' no acepta ningún complementador oracional como si, que, aunque, etc. La imposibilidad de complementadores oracionales en pp' es particularmente interesante porque es uno de los indicios más fuertes de la naturaleza no oracional de este componente;

- otro indicio, igualmente fuerte, de la naturaleza no oracional de este componente es la imposibilidad de que en la frase participial absoluta aparezcan dos SN no correferenciales, aun en el caso de un verbo transitivo: *entregados los equipajes Maria . . . . o *entregados María los equipajes, . . o * María entregados los equipajes, . . .

(Quiero en cambio adelantar - aunque aquí sea sólo en forma parentética - que una expansión posible de pp' consiste en 
agregarle un lugar nominal apropiado para dar cuenta también de las frases participiales absolutas del italiano en que aparece, junto con el SN, también una partícula reflexiva, como muestran los ejemplos 4):

4) a. Presentatasi Maria, cominció a piovere.

b. Rottasi la tazza, ho fatto a meno del caffé.

La forma más reducida de pp' es 5):

5)

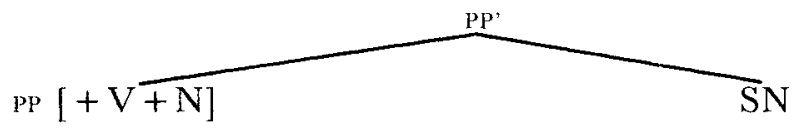

Veamos ahora la utilización de pp' en el análisis de las construcciones ejemplificadas en 3).

\section{La frase participial absoluta con participio pasado transitivo}

Empiezo con la frase participial absoluta porque es la construcción sintáctica en que más clara y directamente aparecen el pp' y sus características básicas. Considérense los ejemplos 6):

6) a. Entregados los equipajes, empezó la huelga.

b. *Entregados, empezó la huelga.

c. *Entregado los equipajes, empezó la huelga.

d. *Entregados equipajes, empezó la huelga.

e. Una vez entregados los equipajes, empezó la huelga.

f. * Si entregados los equipajes, empezará la huelga.

g. *Que entregados los equipajes, empezó la huelga.

h. *Aunque entregados los equipajes, empezó la huelga.

Los ejemplos 6) reflejan las características básicas del pp' que he mencionado anteriormente. El 6)a muestra además que el pp' que constituye la frase participial absoluta carece de cualquier relación sintáctica directa con elementos de la oración externos a ella. Esta observación es relevante en particular por lo que se refiere a la discusión acerca de la interpretación del "sujeto" de la frase participial absoluta: en empezó la huelga no hay ningún sujeto posible para entregados los equipajes. Subrayo este aspecto porque sería muy fácil interpretar que la frase participial absoluta tiene un sujeto externo a ella en ejemplos como 7): 
7) Entregados los equipajes, María se fue a la cafetería.

Esta oración se presta, efectivamente, a la interpretación de que María es el sujeto del pp. Sin embargo, no hay ninguna razón sintáctica que obligue a esta interpretación, como no hay ninguna que impida interpretar que, en 7), los que entregaron los equipajes fuimos, por ejemplo, nosotros: la oración sería perfectamente adecuada para decir que, después que nosotros entregamos los equipajes, María se fue a la cafetería (y yo fui a comprar cigarros). Lo que dice 7) es que fueron entregados los equipajes, sin ninguna especificación acerca de quién efectuó la operación, lo que permite - pero no obliga- a interpretar que fue María, o nosotros o alguien, según las circunstancias contextuales.

Tampoco 8)

8) Entregados los equipajes, María los olvidó

muestra una relación necesaria y unívoca entre el clítico objeto directo y el SN del pp': en esta oración el clítico es un deíctico, sin una relación sintáctica necesaria con el SN del pp'. Puede referirse a los equipajes, como a cualquier otro elemento masculino plural presente en el contexto. Por ejemplo, María puede haber olvidado a los maleteros que han traído los equipajes hasta el mostrador, como resulta claro en 9):

9) Entregados los equipajes, María los olvidó y no les pagó ni un centavo.

La inserción de la frase participial absoluta, analizada en términos de pp', en la configuración global de la oración no me parece particularmente problemática. En primera aproximación sugiero 10):

10)

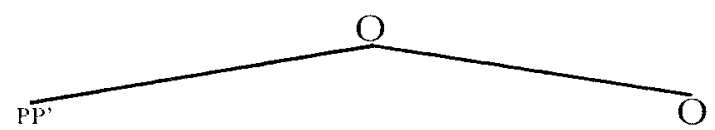

Esta configuración da cuenta de la ausencia de interrelación sintáctica entre pp' y $\mathrm{O}$ y del hecho de que la frase participial absoluta ocupa el lugar más a la izquierda de la oración.

Son pp' también las frases participiales absolutas del italiano y del francés: las diferencias que hay entre las tres lenguas son 
compatibles con este análisis. Debo señalar, sin embargo, que hasta ahora no tengo explicación para una de las diferencias: en francés el SN de pp' no puede aparecer, en la oración superficial, a la derecha del pp y aparece siempre a su izquierda, como se muestra en el contraste de 11):

11) a. Les lettres écrites, Jean est parti.

b. *Écrites les lettres, Jean est parti.

Sin embargo, se puede sostener fácilmente que también este SN está generado a la derecha del núcleo, como en español y en italiano, por paralelismo con el SV correspondiente Jean a écrit les lettres. No tengo por el momento ninguna explicación de esta anomalía, para cuyo esclarecimiento habrá tal vez que tomar en consideración que el francés, al contrario del español y del italiano, no es una lengua pro-drop. Pero, ¿cuál sería la posible relación entre el SN que aparece a la izquierda del pp en la estructura superficial de la frase participial absoluta y la ausencia de la característica pro-drop? Ésta es una de las preguntas que quedan pendientes.

\section{Frase participial absoluta con verbos intransitivos}

Hasta ahora he hablado solamente de frases participiales absolutas en que aparece un pp de verbo transitivo. El análisis de pp' con pp de verbos intransitivos aporta un dato en particular que merece mucha atención: hay dos tipos diferentes de verbos intransitivos. El verbo intransitivo típico, por ejemplo mentir y toser, no puede aparecer en frase participial absoluta, ni en español, ni en italiano, ni en francés, y esto está evidentemente relacionado con el hecho de que los verbos intransitivos son tales precisamente porque no generan ningún $\mathrm{SN}$ a su derecha. Hay, sin embargo, una clase de verbos "intransitivos" que aparecen en frases participiales absolutas, como muestro en 12):

12) a. Llegada María, se fue la luz.

b. Muerta mi abuela, heredé una fortuna.

c. *Mentida María, se averiguó la verdad.

d. *Mentida la noticia, se averiguó la verdad.

e. ${ }^{*}$ Tosida la niña, llamamos al doctor. 
Para el italiano, que es la lengua más rica respecto a esta estructura en particular, hay muchos datos que comprueban que se trata de verbos ergativos —o sea de verbos que generan el "sujeto" a su derecha- porque se comportan en muchos aspectos de una manera diferente de los verbos intransitivos. En español, en cambio, no he encontrado evidencias de ergatividad, salvo precisamente ésta: hay pp "intransitivos" que sin embargo aparecen en pp', lo que - según mi análisis - implica necesariamente que generan un $\mathrm{SN}$ a su derecha. En el caso específico de estos verbos el $\mathrm{SN}$ generado a su derecha sólo puede ser su sujeto. Si deducimos de este análisis que también en español hay ergativos, que serían precisamente los intransitivos que pueden aparecer en pp', tendríamos entonces una explicación de por qué hay una diferencia importante entre 13) y 14):

13) Llegó María.

14) Mintió María.

La 13) suena completamente normal y no marcada con el sujeto a la derecha del verbo, mientras que la 14) no puede ser considerada la forma básica correspondiente a María mintió.

En español contemporáneo el fenómeno de la ergatividad, si existe, es muy poco visible porque los verbos que serían ergativos según este análisis no manifiestan otras diferencias sintácticas respecto a los intransitivos "normales", aparte de que su sujeto suena perfectamente bien y no marcado cuando aparece a su derecha, como en 13). Me parece, sin embargo, que no se debe subevaluar el contraste ejemplificado en 12), manteniendo presente la posibilidad de que este indicio conduzca a encontrar otras pruebas de ergatividad en la lengua.

Si se acepta el razonamiento expuesto hasta aquí, llegamos inevitablemente a la siguiente conclusión:

15) El sintagma participial, pp', es intrínsecamente una estructura ergativa, compuesta por un núcleo que acepta y requiere un solo $\mathrm{SN}$, generado a su derecha.

Se puede considerar provisionalmente no pertinente que este SN pueda ser analizado como sujeto o como objeto directo del verbo cuando éste aparece conjugado en una oración (llegada María vs. María llegó; entregados los equipajes vs. alguien entregó los equipajes o los equipajes fueron entregados). Este dato tomará un lugar importante, en cambio, al momento de analizar la asignación de caso al SN: en italiano hay evidencias morfológicas de que éste puede 
tener el caso nominativo o el acusativo, y el contraste sugiere fuertemente - pero no necesariamente- la posibilidad de analizar el SN de pp' en términos de sujeto u objeto directo del pp.

En lo que sigue de esta sección voy a sostener que lo que aparece en los ejemplos 3)c, 3)d, 3)e y 3)f es este mismo pp'. Las modificaciones a que está sometido en estos casos son las necesarias para su inserción en la estructura de la oración y pueden explicarse sin alterar sustancialmente las características definitorias de su estructura básica.

\section{El verbo tener con sintagma participial}

La relativa pobreza del español en frases participiales absolutas está ampliamente compensada por la existencia de oraciones con tener, en las cuales el pp' aparece con mucha simplicidad y evidencia. Tener no tiene correspondiente ni en italiano ni en francés, por lo que se refiere a su comportamiento respecto a $\mathrm{pp}$ ': lo que mejor le corresponde es habeo en latín. Examínense los ejemplos 16) y 17):

16) a. Tengo traducidos los resúmenes.

b. Tengo pintadas las sillas.

17) a. Tengo una blusa bordada.

b. Tengo un gato siamés.

El tener de los dos ejemplos no es el mismo: el de 17) es un verbo transitivo cualquiera y aquí solamente nos concierne el tener del ejemplo 16). Me parece que 18) da cuenta de la manera más simple y más adecuada de su estructura:

18)

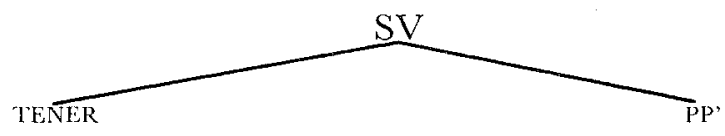

El pp' que aparece con tener, sin embargo, nos muestra que su estructura interna debe ser más compleja de la indicada en 5). En efecto, debemos dar cuenta del hecho de que el SN del pp' puede moverse también a la izquierda del pp, dando lugar a las oraciones 19): 
19) a. X Tengo los resúmenes traducidos.

b. X Tengo las sillas pintadas.

Cada oración obtenida por medio de este movimiento resultará siempre ambigua porque su estructura superficial será idéntica a la estructura de cualquier oración transitiva cuyo objeto directo contenga un modificador. Es evidente, sin embargo, que 19)a y 19)b son, cada una, la estructura superficial de dos oraciones distintas, porque así lo indican tanto el mero hecho de su ambigüedad como una serie de hechos sintácticos que permite diferenciar las dos oraciones distintas. Para indicar sólo uno, nótese que si queremos sustituir el SN con un clítico tendremos que a cada una de las dos interpretaciones de 19)a y de 19)b corresponderán dos diferentes oraciones con clítico; en 20) aparecen las oraciones que corresponden a la interpretación de la estructura tener $+\mathrm{pp}$ ' y en 21) las que corresponden a la interpretación de tener + objeto directo:

20) a. Los tengo traducidos.

b. Las tengo pintadas.

21) a. Los tengo.

b. Las tengo.

Volviendo a la estructura interna de pp', entonces, debemos aceptar la necesidad de enriquecerla para dar cuenta de la posición nominal en la cual se ha movido el SN de las 19), a la izquierda del pp (y ya en el ejemplo 4 he señalado otra evidencia independiente de que el pp' no puede contener sólo un lugar para $\mathrm{SN}$ ).

Resulta extremadamente interesante constatar que pp' no puede contener sólo un lugar para SN pero, al mismo tiempo, no puede contener dos SN diferentes, en el sentido de que sean SN con referentes distintos. Este rasgo peculiar de la estructura interna de pp' adquiere particular evidencia y significación en el análisis de las estructuras de ser, essere, être + pp'.

Ser, essere, être con sintagma participial

Incluyo aquí esta sección solamente para mostrar qué diseño y alcance de la investigación sugiere el desarrollo de mi hipótesis acerca de los pp del español, italiano y francés. Los pp' que apa- 
recen con la cópula y en las estructuras predicativas requieren en realidad ser tratados de una manera mucho más extensa y detallada y presentaré aparte un trabajo dedicado a estas construcciones en particular. Lo que me interesa destacar aquí es que me está resultando sostenible y plausible que el pp' se inserte en las oraciones copulativas y en el nodo de los complementos predicativos. Nótese que por "oración copulativa" entiendo cualquier oración en que aparezca ser, essere o être. Son, por lo tanto, ejemplos de oraciones con inserción de pp', según mi hipótesis, todas las que aparecen con ser, essere, être en 22), 23), 24), 25), 26) y 27); las que aparecen con avere y avoir están para marcar contrastes significativos por lo que se refiere a las estructuras muy específicas en que aparece la cópula:

22) a. Fueron aceptados todos los alumnos.

b. Todos los alumnos fueron aceptados.

23) a. É' arrivata Maria.

b. Maria é arrivata.

c. Marie est arrivée. ( ${ }^{*}$ Est arrivée Marie.)

24) a. Maria ha lavato i piatti.

b. Maria ti ha lavato i piatti.

c. Maria si é lavata i piatti.

25) a. Maria e Carla hanno incontrato il professore.

b. Maria e Caria si sono incontrate.

26) a. Marie a lavé les assiettes.

b. Marie t'a lavé les assiettes.

c. Marie s'est lavée.

27) a. Marie et Sara ont rencontré les journalistes.

b. Marie et Sara se sont recontrées.

En estos ejemplos podemos diferenciar por un lado las pasivas y las ergativas de 22) y 23) y por el otro las reflexivas y recíprocas de 24)c, 25)b, 26)c y 27)b. Para dar cuenta de las primeras podría parecer suficiente un pp' que tenga un solo lugar para nominal, pero, tomando en consideración las reflexivas y las recíprocas, constatamos la necesidad de, por lo menos, dos lugares para nominal: un lugar para $\mathrm{SN}$ y otro para la partícula reflexiva o recíproca. Lo peculiar es que se trata de dos nominales que tienen la característica intrínseca de correferir: en términos intuitivos, es un solo nominal "colado"' en dos lugares diferentes de la estructura. Por otra parte debemos mantener que el SN de pp' se genera en todos los casos a la derecha del pp, y por lo tanto hay un lugar nominal más, que puede estar vacío, también en 
esta posición. Vemos entonces 1): varios lugares nominales y 2): que todos estos lugares nominales pueden en realidad estar ocupados por sólo un SN: nunca efectivamente pueden aparecer en estas estructuras dos $\mathrm{SN}$, que sean dos en el sentido estricto de que tengan dos referentes distintos o distinguibles. Las características 1) y 2), juntas, sugieren fuertemente un análisis en términos de cadena nominal: la inserción de pp' es posible en una oración con ser, essere o être porque también la cópula tiene la peculiaridad de generar una cadena nominal. Los dos segmentos de cadena nominal, el de la cópula y el del pp', podrán soldarse entre sí y formar una sola cadena nominal, a condición de que compartan el mismo SN, o sea cada vez que el SN del uno y del otro sean el mismo. Esto explicaría, me parece, el mecanismo de la coexistencia de cópula y pp'.

\section{Los complementos predicativos del sujeto y del objeto directo}

Vemos ejemplos de predicativos del sujeto y del objeto respectivamente en 28) y 29):

28) Los resúmenes llegaron traducidos.

29) a. X Entregaré los resúmenes traducidos.

b. Los entregaré traducidos.

La 29)a es ambigua porque en una de sus interpretaciones el pp es un modificador, análogamente a lo que hemos visto anteriormente en el ejemplo 19). La interpretación que le corresponde al complemento predicativo del objeto es la que la oración tiene en 29)b.

Quiero sostener que los predicativos del sujeto y del objeto tienen como estructura básica el mismo pp' que hemos visto hasta ahora, con la particularidad "predicativa"' de que el SN generado a la derecha del pp en pp' no aparece nunca en su lugar de origen, porque aparece en el lugar del sujeto o del objeto directo de la oración. Esta peculiaridad es precisamente la que me sugiere que también los pp' predicativos deberán ser analizados en términos de cadena nominal: la cadena comprenderá, naturalmente, el SN sujeto u objeto directo y el lugar vacío del SN a la derecha de pp. Un rasgo particularmente significativo de los predicativos es que no aparecen nunca, en la misma oración, un predicativo del sujeto y un predicativo del objeto: si hay predicativo del suje- 
to no hay predicativo del objeto, y viceversa. Esta imposibilidad de coexistencia de los dos predicativos debe ser explicada, y supongo que tiene una relación con el lugar de inserción del predicativo en la estructura global de la oración. ¿Habrá en ésta sólo un lugar para predicativos? Mi intuición es que éstos son en algún sentido simétricos con las frases participiales absolutas, y así como hay un solo lugar para frases participiales absolutas y este lugar parece muy alto, tal vez hay un solo lugar para predicativos, igualmente alto en la estructura. Por el momento no puedo ofrecer más que intuiciones a este respecto.

\section{EL PARTICIPIO PASADO SIN CONCORDANCIA NOMINAL}

Para dar cuenta del pp sin concordancia nominal me parece que la solución más sencilla es acudir al concepto de parametrización. $\mathrm{Si}$, como he expuesto en la premisa, el pp está inherentemente categorizado como $[+\mathrm{V} \pm \mathrm{N}]$, podemos hacer la hipótesis de que el latín no tenía activado el conjunto de rasgos $[+\mathrm{V}-\mathrm{N}]$ y que este conjunto ha sido elicitado como parte del cambio del latín a las lenguas romances actuales. Dicho de otra manera, el surgimiento del pp invariable en español, italiano y francés correspondería a la activación del parámetro $[+\mathrm{V}-\mathrm{N}]$. Los datos muestran en efecto que el pp que carece de concordancia nominal, que no existía en latín, se ha ido afirmando paulatinamente en el curso del desarrollo de las lenguas romances, sustituyendo a un número siempre mayor de pp con concordancia nominal.

En español contemporáneo, la distribución entre pp con concordancia nominal y op invariable ha llegado a ser tan consistente y regular que casi hace parecer banal el problema: efectivamente, tomando en consideración solamente el español, nunca se me hubiera ocurrido preguntarme cómo ha surgido el pp que no tiene concordancia nominal.

Las evidencias de que no se trata de un problema banal se encuentran muy claramente en los datos del italiano y del francés. En italiano, por ejemplo, tenemos 30):

30) a. Maria ha mangiato le mele.

b. Le mele che Maria ha mangiato erano mature.

c. Maria le ha mangiate.

En 30)a y b el pp no puede concordar nominalmente con nin- 
gún elemento de la oración pero en 30)c debe concordar obligatoriamente con el clítico objeto directo.

En italiano y en francés la distribución de los dos tipos de pp está lejos de ser tan simple como ha llegado a ser en español y para estas lenguas, por lo tanto, el problema de la presencia o ausencia de la concordancia nominal en el pp es muy llamativo. Por otra parte, una vez percibido el problema, resulta inmediatamente evidente que también en etapas anteriores del español debía haber una distribución diferente de la actual, con la línea de evolución muy clara: poco a poco deben haber aumentado los casos en que un pp que concordaba nominalmente se sustituía por un pp invariable. Nunca me he ocupado hasta ahora de la evolución del español (ni de ninguna otra lengua) y por lo tanto no pretendo dar una descripción de las etapas de este cambio del pp. Me limito a afirmar que este cambio paulatino se puede deducir del análisis en términos de parametrización que estoy proponiendo. Para tener una confirmación aunque fuera de lo más superficial, he ojeado algunas gramáticas del español y he encontrado de inmediato "el antiguo había ganada la batalla" (en lugar del actual habia ganado la batalla). Aparte de esta confirmación, que he encontrado en la p. 300 de la Gramática histórica española de Vicente García de Diego², varios hispanistas me han confirmado en conversaciones informales la paulatina sustitución de pp con concordancia nominal por pp invariable. Cerrada esta breve y superficial excursión en el campo de la lingüística histórica, vuelvo ahora a la hipótesis que estoy proponiendo.

La descripción de los casos sintácticos en que el pp deja de manifestar concordancia nominal resulta muy compleja para el italiano y el francés, en los que el proceso no ha llegado todavía a la distribución más compacta que tiene el español. En esta última lengua, en efecto, la distribución es actualmente muy precisa: el pp aparece sin concordancia nominal siempre y sólo cuando está asociado con el auxiliar haber, y no he podido construir ningún contraejemplo a esta afirmación. Este pp es el que corresponde a la activación del conjunto de rasgos $[+\mathrm{V}-\mathrm{N}]$. Su morfología, sin embargo, no tiene la posibilidad de manifestar las oposiciones pertinentes para realizar características fundamentales del verbo como tiempo y concordancia de persona y la función del auxiliar es precisamente la de especificar estos rasgos verbales por

2 Vicente García de Diego, Gramática histórica española, 3a. ed. corregida, Gredos, Madrid, 1970. 
medio de su conjugación. El complejo constituido por las formas conjugadas de haber más el pp $[+\mathrm{V}-\mathrm{N}]$ es entonces solamente una de las alternativas morfológicas de la conjugación del verbo. Éste, naturalmente, no tiene ninguna concordancia de tipo nominal, o sea, concordancia de género y número. Entonces, así como hay ausencia de cualquier concordancia de tipo nominal en el SV de 31)a:

31) a. Pedro comió las manzanas.

tendremos ausencia de concordancia nominal en el SV de 31)b:

31) b. Pedro ha comido las manzanas.

El resultado del surgimiento del pp $[+\mathrm{V}-\mathrm{N}]$ es un enriquecimiento de la conjugación verbal. La configuración básica que le corresponde, según este análisis, es 32):

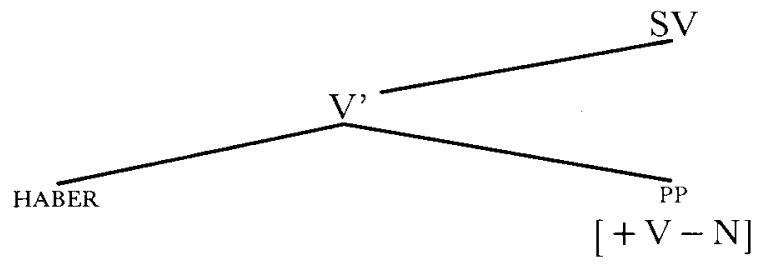

El V' que propongo en 32) me parece adecuado para todo tipo de pp del español que aparezca con haber y todo tipo de pp que aparezca sin concordancia nominal: los transitivos, los intransitivos, los reflexivos, los recíprocos y los impersonales. (Las peculiaridades de estos diferentes tipos de verbos se manifestarán en el ámbito del SV y, por ejemplo, habrá SN en el SV de los verbos transitivos y no lo habrá en el SV de los intransitivos.) V' expresa entonces la coexistencia de haber y del pp invariable: cada vez que aparece un pp sin concordancia nominal, estará asociado con haber y cada vez que aparece haber con un pp, éste carecerá de concordancia nominal, independientemente del tipo de verbo de que se trate y de cualquier otro rasgo sintáctico peculiar de la oración. En los ejemplos 33) he tratado de reflejar una variedad suficientemente amplia de tipos de verbo y de acontecimientos sintácticos:

33) a. María ha leído las revistas.

b. María las ha leído. 
c. Las revistas que María ha leído...

d. ¿Cuáles revistas ha leído María?

e. María ha mentido.

f. Ha llegado María.

g. María se ha lavado.

h. María se ha lavado las manos.

i. María se ha desmayado.

j. La taza se ha roto.

k. María y Juana se han encontrado.

l. Ayer ha llovido.

m. María ha parecido culpable.

n. El tribunal ha juzgado culpable a María.

El patrón tipológico del español es que los elementos generados a la izquierda del núcleo del sintagma son especificadores mientras que los elementos generados a la derecha son complementos. En la interpretación de V' por el momento me inclino a pensar que se trata de una proyección del pp, y entonces haber resultaría ser un especificador. Me parece menos interesante la posibilidad de que el V' sea proyección de haber, en cuyo caso el participio pasado resultaría ser un complemento de éste.

En italiano y en francés este proceso no ha llegado todavía a su cumplimiento, pero resulta evidente que ésta es la dirección de su evolución: V' sustituirá por completo a pp' con avere y avoir. Por ejemplo, en italiano podemos todavía ver un pp obligatoriamente $[+\mathrm{V}+\mathrm{N}]$ (o sea con concordancia nominal) cuando el objeto directo precede al verbo bajo forma de clítico de tercera persona, como aparece en los ejemplos 34 ):

34) a. Gianni le ha viste.

b. ${ }^{*}$ Gianni le ha visto.

Sin embargo, como ha subrayado L. Burzio en Italian syntax ${ }^{3}$, la alternancia es posible con los clíticos de primera y segunda persona, singular y plural, tal como se ve en 35 ):

35) a. Gianni vi ha visti.

b. *Gianni vi ha visto.

Esto muestra que ya aparece un V' también en esta configuración sintáctica particular, a pesar de que contenga avere. La pre-

${ }^{3}$ L. Burzio, Italian syntax, D. Reidel, Dordrecht, 1986. 
dicción, naturalmente, es que 35)b reemplazará a 35)a y que, más tarde, también 34)b reemplazará a 34)a. (Por lo que se refiere a una posible justificación de que el proceso sea más avanzado con los clíticos de primera y segunda persona, parece razonable la consideración de Burzio de que "a diferencia de la forma de tercera persona, los clíticos objeto directo de primera y segunda persona no están diferenciados por lo que se refiere al género'”.)

El francés, respecto al italiano, presenta aún un mayor número de casos sintácticos en que todavía no se integra a la conjugación del verbo el componente $V^{\prime}$ : por ejemplo, además del clítico objeto directo, también el objeto directo realizado como pronombre relativo implica la concordancia nominal del pp que aparezca en el SV. Así, en francés, hay no sólo el contraste ejemplificado en 36), sino también el de 37):

36) a. Jean les a écrites.

b. ${ }^{*}$ Jean les a écrit.

37) a. La lettre que Jean a écrite arrivera demain.

b. ${ }^{*}$ La lettre que Jean a écrit arrivera demain.

En todo caso me parece que las pruebas son suficientes para afirmar que los verbos haber, avere y avoir se han especializado, perdiendo fundamentalmente la característica de habeo de aparecer con pp', salvo en un reducido número de casos del italiano y del francés en que todavía la mantienen, aunque diacrónicamente van disminuyendo.

\section{Goncluesión}

Como ya dije, este trabajo es un adelanto de los resultados del análisis de los participios pasados del español, italiano y francés. Falta tratar puntos importantes como, por ejemplo, los problemas relativos a la asignación de caso al SN del sintagma participial, el participio pasado asociado con estar y muchos aspectos de pp' y V' que son ciertamente cruciales para una comprensión más detallada y precisa de los fenómenos considerados. Además, apenas he esbozado el análisis en términos de cadena nominal para la inserción de los sintagmas participiales en las oraciones copulativas y en los complementos predicativos: este tema, por sí solo, amerita por lo menos un artículo aparte.

A pesar de estas limitaciones evidentemente importantes, creo 
sin embargo que esta exposición es pertinente, tanto porque los resultados obtenidos hasta ahora me parecen suficientemente estimulantes y significativos, como por lo útil que resultará abrir el tema a críticas, comentarios y futuros desarrollos.

Bruna Radelli

Instituto Nacional de Antropología e Historia 